\title{
O DSM-5 e suas implicações no processo de medicalização da existência
}

The DSM-5 and its implications in the process of medicalization of existence

EI DSM-5 y sus implicaciones en el proceso de medicalización de la existencia

\author{
Marina Silveira de Resende \\ Samira Pontes ${ }^{* *}$ \\ Roberto Calazans ${ }^{* *}$
}

\begin{abstract}
Resumo
O lançamento da quinta edição do Manual Diagnóstico e Estatístico de Transtornos Mentais (DSM), fruto de um demorado processo de revisão do DSM-IV-TR, acarretará mudanças na estrutura do próprio manual, que passará de um modelo de diagnóstico categorial para um modelo longitudinal. Tais mudanças proporcionarão um caráter preditivo aos diagnósticos. Nosso objetivo é discutir as implicações de algumas novas categorias diagnósticas propostas pelo DSM-5, no que tange, principalmente, ao fortalecimento do movimento de medicalização da existência. Apontamos como um dos principais desencadeadores dessa medicalização o fato de que os DSM deixam de ter um uso exclusivamente clínico. Para isso, apresentamos o aumento do número de vendas de medicamentos psiquiátricos no Brasil como um indicador desse movimento de medicalização da existência.
\end{abstract}

Palavras-chave: Clínica. Medicalização. DSM-5. Diagnóstico.

\begin{abstract}
The release of the fifth edition of the Diagnostic and Statical Manual of Mental Disorders (DSM), the fruit of a long revision process of the DSMIV-TR will result in changes in the structure of its own manual, which will change from a categorical diagnostic model to a longitudinal model. Such changes will confer a predictive characteristic to diagnosis. Our objective is to discuss the implications of some new diagnostic categories proposed by the DSM-5, concerning, especially, the strengthening of the movement that medicalizes its own existence. We point to, as one of the main triggers of
\end{abstract}

\footnotetext{
Texto submetido em 11 de março de 2013 e aprovado para publicação em 28 de agosto de 2014.

Mestra pelo Programa de Pós-Graduação em Psicologia da Universidade Federal de São João del-Rei (UFSJ), psicóloga pela UFSJ, psicóloga do Instituto de Ensino Superior Presidente Tancredo de Almeida Neves (IPTAN). Endereço: Praça Dom Helvécio, n. 74 - Fábricas, São João del-Rei-MG, Brasil. CEP: 36301-160. E-mail: ninasresende@gmail.com.

** Mestranda do Programa de Pós-Graduação em Psicologia da UFSJ, psicóloga pela UFSJ, bolsista CAPES. Endereço: Praça Dom Helvécio, n.o 74 - Fábricas, São João del-Rei-MG, Brasil. CEP: 36301-160. E-mail: spcpontes@gmail.com.

${ }^{* * *}$ Doutor em Teoria Psicanalítica pela Universidade Federal do Rio de Janeiro, professor associado 2 da UFSJ e do Programa de Pós-Graduação em Psicologia da UFSJ, bolsista Produtividade em Pesquisa nível 2 CNPq. Endereço: Praça Dom Helvécio, n. ${ }^{\circ}$ 74 - Fábricas, São João del-Rei-MG, Brasil. CEP: 36301-160. E-mail: roberto.calazans@gmail.com.
} 
this medicalization, the fact that the DSMs are no longer exclusively used clinically. To that end, we present the raise in the number of psychiatric drug sales in Brazil, as an indicator of this movement of medicalization of the existence.

Keywords: Clinic. Medicalization. DSM-5. Diagnosys.

\section{Resumen}

La publicación de la quinta edición del Manual Diagnóstico y Estadístico de los Trastornos Mentales (DSM), producto de un lento proceso de revisión del DSM-IV-TR, implicará cambios en la estructura del propio manual, que pasará de un modelo de diagnóstico categorial a un modelo longitudinal. Tales cambios proporcionarán un carácter predictivo a los diagnósticos. Nuestro objetivo es discutir las implicaciones de algunas nuevas categorías diagnósticas propuestas por el DSM-5, en lo que dice respecto, principalmente, al fortalecimiento del movimiento de medicalización de la existencia. Apuntamos como uno de los principales desencadenadores de esta medicalización el hecho que los DSM dejaron de tener un uso exclusivamente clínico. Para eso, presentamos el aumento del número de vendas de medicamentos psiquiátricos en Brasil como un indicador de este movimiento de medicalización de la existencia.

Palabras clave: Clínica. Medicalización. DSM-5. Diagnóstico.

\section{Introdução}

Dublicado pela Associação Americana de Psiquiatria (APA), o Manual Diagnóstico e Estatístico de Transtornos Mentais (DSM) é o dispositivo oficial de traçar os diagnósticos psiquiátricos nos Estados Unidos, sendo utilizado em grande escala no mundo e, tendo assim, grande influência sobre a Classificação Internacional de Transtornos Mentais da Organização Mundial de Saúde (OMS). Além de ser usado por profissionais da área clínica, o DSM visa a ser incorporado globalmente em outras áreas de atuação, tais como a jurídica, escolar e organizacional.

O DSM, principalmente a partir de sua terceira versão (Associação Americana de Psiquiatria [APA], 1980), foi elaborado com a pretensão de ser um instrumento científico e principalmente ateórico de diagnóstico. Ou seja, a perspectiva era de que esse manual conseguisse ultrapassar as diversas teorias que existem no campo da psicopatologia, de modo a unificar os sistemas diagnósticos no campo do sofrimento psíquico. Mas como realizar essa tábula rasa se a própria história da 
psicopatologia é de debates, composta essencialmente por teorias que divergem entre si?

Os DSM partem de uma perspectiva teórica pragmática, em que o sujeito deve estar no melhor funcionamento social possível:

O invisível aos olhos, mas que habita na língua do DSM, é o consenso sobre a "ordem" que a sociedade espera dos filhos das famílias "normais": que sejam bons, carinhosos, tranquilos, adaptados, que aprendam bem "suas" lições, que comam e durmam bem e que, chegando o momento, estejam dispostos a defender até a morte, na guerra ou na paz, os valores do sistema. [...] A norma, a ordem, a média não explícita é a (classe) média, "modelo", cujos filhos não devem ser "diferentes" dos pais, moldados pelo american-way-of-life. (Jerusalinksy \& Fendrik, 2011, p. 34)

No entanto, como diz a premissa foucaultiana: "Onde há poder, há resistência" (Foucault, 1988, p. 91), em 2011, foi lançado o Movimento Internacional Stop-DSM, uma iniciativa que tem como objetivo a crítica ao critério único de diagnóstico clínico proposto pelo DSM. Esse movimento propõe um questionamento em relação à lógica classificatória do DSM, defendendo, em contrapartida, uma abordagem subjetiva e clínica do sofrimento psíquico. (Lanaspa, 2014).

O Stop-DSM conta com a adesão de diversos profissionais, grupos e instituições ao redor do mundo que, implicados com as consequências do desenvolvimento de novas categorias diagnósticas do DSM-5, elaboraram manifestos para recolher assinaturas e apresentá-las à Organização Mundial da Saúde. Este trabalho é decorrência desse movimento de crítica que ainda perdura.

O manual está em sua quarta versão, DSM-IV-TR (APA, 2002), e é previsto para o primeiro semestre de 2013 o lançamento de sua quinta versão. $O$ advento do DSM-5 acarretará mudanças estruturais no próprio manual que darão um caráter preditivo aos diagnósticos. Não obstante, tais mudanças atravessarão o campo conceitual, passando a fortalecer o movimento já orientado pelos DSM de patologização e medicalização da vida dos sujeitos. Pretendemos, desse modo, refletir criticamente o projeto do DSM-5 e suas implicações na criação de novas epidemias de transtornos mentais. Apresentaremos também a velha relação dos DSM e a indústria farmacêutica, focando no aumento das vendas de medicamentos psiquiátricos no Brasil. 


\section{Os DSM, a farmacologia e a realidade brasileira}

O consenso acerca do conceito de saúde mundialmente aceito, responsável por guiar esse ambicioso projeto da psiquiatria moderna, decorre de uma organização política: a OMS.

Segundo a constituição da OMS, "a saúde de todos os povos é essencial para conseguir a paz e a segurança, e depende da mais estreita cooperação dos indivíduos e dos Estados" (Organização Mundial de Saúde [OMS], 1946). Dessa forma, o conceito de saúde foi definido como "Um estado de completo bem-estar físico, mental e social, e não consiste apenas na ausência de doença ou de enfermidade" (OMS, 1946). Ou seja, para interagir satisfatoriamente com a sociedade, por meio do trabalho e da família, o homem precisa estar dotado de um pleno bem-estar. Daí vemos que a saúde é definida também como a total ausência de síndromes psicopatológicas (Hansen, 2004).

Tal perspectiva não deixa de ter consequências na prática clínica. O DSM-IVTR (APA, 2002), edição atual do manual, é responsável pela produção de pelo menos três "epidemias" de transtornos mentais:

a) transtorno bipolar;

b) transtorno de déficit de atenção e hiperatividade; e

c) autismo infantil (Fendrik \& Jerusalinsky, 2011). Essa produção se dá pelos critérios diagnósticos que consideram somente os fenômenos sem levar em conta a estrutura de organização destes.

Junto com essas epidemias, temos também dados que apontam o aumento do número de prescrição de medicamentos no Brasil (Brasil, 2008), sendo esse aumento um indicador da associação estabelecida entre psiquiatria e farmacologia, ou seja, entre a ideia de cura e a ideia de supressão de sintomas.

Dados do governo (Brasil, 2011) mostram que 44\% dos remédios controlados vendidos em farmácias e drogarias no Brasil são para o tratamento de transtornos mentais e de comportamento, sendo os ansiolíticos os mais consumidos no País nos anos de 2007 a 2010.

Somando a venda de Clonazepam (Rivotril), Bromazepam (Lexotan) e Alprazolam, encontramos um total de 19,3 milhões de caixas vendidas. Já a venda do antidepressivo Fluoxetina atingiu, em 2011, a margem de 3,5 toneladas. 
Em relação à venda do Metilfenidato, medicamento receitado para o TDAH (transtorno de desatenção e hiperatividade), tivemos no Brasil, entre os anos 2000 e 2004, o aumento de $1020 \%$ de vendas de caixas e, entre 2004 e 2008, outro aumento de $930 \%$ na venda do produto:

\begin{abstract}
A venda crescente de medicamentos tem gerado, inclusive, distorções no meio médico, pois muitos desses profissionais vêm recebendo "brindes" dos laboratórios pela quantidade de remédios de determinada marca que receitam a seus pacientes. A pressão dos laboratórios é tão evidente que, em 2010, o Conselho Federal de Medicina proibiu os médicos de receberem "vantagens materiais" por receitarem determinados medicamentos e voltou atrás em 2012, permitindo que fosse possível oferecer, em troca, uma viagem para Congresso por ano, financiada por determinado laboratório, justificando que é uma "tendência mundial" (Conselho Federal de Psicologia [CFP], 2012, p. 5)
\end{abstract}

Os dados de venda de medicamentos no Brasil apontam para um acontecimento de âmbito mundial: de medicalização do sofrimento. Desse modo, devemos ver que implicações teria o advento do DSM-5 nesse movimento medicalizante.

Para se ter uma ideia da gravidade da medicalização da subjetividade do homem, ou seja, da apropriação pelo campo médico de questôes que anteriormente não eram considerados médicas, mas de outra ordem, como jurídica, social ou existencial, hoje podemos encontrar mais de 300 categorias oficiais, em um aumento exponencial desde que se passou a usar a estatística e não a clínica como método de definição de categorias. Com o novo manual, o DSM-5, esse número tende a aumentar.

Com essa apropriação, a psiquiatria biologizante estabelece um discurso único, uma relação de poder em que o médico é detentor de uma verdade a despeito do sujeito e que atua na promoção de uma ordem social sem sintomas.

Os impactos do aumento das categorias e critérios diagnósticos são vários. No Brasil, uma recente pesquisa (Andrade et al., 2012) sobre a saúde mental dos moradores da metrópole de São Paulo, que teve como base os critérios diagnósticos presentes no DSM-IV-TR (2002) e no CID-10, demonstrou que cerca de $30 \%$ da sua população sofre de algum transtorno psiquiátrico, sendo que 1 em cada 16 (5,9\%) entrevistados apresentaram comorbidades de 2 transtornos e 5,8\% dos entrevistados apresentavam comorbidade de 3 ou mais transtornos mentais. 


\section{$\mathrm{O}$ advento do DSM-5 e suas implicações}

As análises dos dados acima deveriam servir para que os revisores do manual agissem com cautela em uma próxima empreitada. No entanto, a APA prepara com entusiasmo o DSM-5, uma tentativa de produzir uma mudança de paradigma nos diagnósticos psiquiátricos.

O modelo adotado nas últimas revisões, o de diagnóstico categorial, será substituído pelo modelo dimensional (APA, 2013), o qual tem como consequência a predição de transtornos mentais (Lucchelli, 2007). Para atingir essa meta, o manual parece relativizar os sinais e sintomas de um transtorno.

Allen Frances, presidente da força-tarefa que trabalhou na revisão do DSMIV-TR (APA, 2002) e atualmente maior crítico do DSM, cita na reportagem Openig Pandora's box (Frances, 2010), algumas das graves mudanças na nova edição do manual, após análise do rascunho que já está disponível na internet (http://www.dsm5.org). Entre elas, estão: escrita pobre e inconsistente, aumento significativo no número de transtornos, diminuição das especificidades para traçar diagnósticos, consequências imprevistas de uso (forense/educação/ organizações).

Entre os novos diagnósticos, Allen Frances (2010) apontou alguns novos transtornos que podem facilmente incluir grande parte da população:

a) Síndrome de risco de psicose: de acordo com Allen Frances (2010), essa é certamente a mais preocupante de todas as sugestôes feitas para o DSM5. De acordo com estudos, da forma que está elaborado no manual, o novo transtorno teria uma taxa de $70 \%$ a $75 \%$ de falsos positivos, taxa susceptível a aumento, uma vez que o diagnóstico será "oficial", de uso geral, podendo se tornar um alvo para as empresas farmacêuticas. Dessa forma, centenas de milhares de adolescentes e jovens adultos receberiam a prescrição desnecessária de antipsicóticos atípicos, sendo que não há prova de que os antipsicóticos atípicos possam prevenir episódios psicóticos. No entanto seus efeitos colaterais são amplamente conhecidos:

$\checkmark$ grande e rápido ganho de peso;

$\checkmark$ estão associados à redução da expectativa de vida (recente alerta da Food and Drug Administration), além de ter um alto custo e estigma.

b) Transtorno depressivo misto de ansiedade: seus sintomas inespecíficos batem com comportamentos que são amplamente observados na 
população em geral e, portanto, imediatamente irá se tornar um dos mais comuns de todos os transtornos mentais em DSM-IV. Naturalmente, a sua rápida ascensão em proporçôes epidêmicas será "competentemente" assistida por marketing farmacêutico. É provável que a medicação não seria muito mais eficaz que o placebo por causa das altas taxas de respostaplacebo em desordens mais leves. Inicialmente colocada como certa a inclusão desse transtorno no DSM-5, hoje ele está sendo repensado, devido à quantidade de críticas enviadas por especialistas aos revisores do manual.

c) Transtorno neurocognitivo menor: é definido por sintomas não específicos de desempenho cognitivo reduzido, que são muito comuns, talvez quase onipresentes, em pessoas acima de 50. Para proteger contra falsos positivos, o manual propóe uma avaliação cognitiva com o objetivo de confirmar que o indivíduo tem diminuição do desempenho cognitivo, mas obter um ponto de referência significativa é impossível na maioria dos casos. Parece que seu limiar foi definido para incluir uma grande parcela da população: $13,5 \%$. Além disso, a sugestão para o teste objetivo provavelmente será amplamente ignorada nos serviços de cuidados primários, nos quais a maior parte do diagnóstico será feita. Medicalizar as deficiências cognitivas esperadas no processo de envelhecimento resultará em tratamentos desnecessários, muitos com medicamentos ineficazes e remédios populares charlatôes.

d) Transtorno de compulsão alimentar periódico: terá uma taxa da população geral estimada em 6\%, e isso provavelmente aumentará quando o diagnóstico se tornar popular e feito em ambientes de cuidados primários. As pessoas que comerem compulsivamente uma vez por semana, durante três meses, de repente terão um "distúrbio mental", sendo submetidas ao estigma e medicamentos com eficácia não comprovada.

e) Transtorno de temperamento disfuncional com disforia: é uma das sugestôes mais mal concebidas pelo DSM-5. A medicalização equivocada de explosões temperamentais. O "diagnóstico" seria muito comum em todas as idades na população em geral e promoveria uma grande expansão no uso de medicamentos antipsicóticos, com todos os riscos descritos acima. Aparentemente, o grupo de trabalho estava tentando corrigir a epidemia de diagnóstico de desordem bipolar na infância, mas sua sugestão é tão mal escrita que não poderia realizar esse objetivo, e, em vez disso, criaria um novo monstro. 
f) "Transtorno parafílico coercitivo": foi inicialmente foi considerado para inclusão no DSM-III-R (sob o nome paraphilic rapism), mas foi rejeitado, pois era impossível estabelecer a diferença, de forma válida e confiável, entre os estupradores cujas ações são o resultado de uma parafilia e a grande maioria dos estupradores que são motivados por outros fatores (como energia). O diagnóstico será inevitavelmente com base apenas no comportamento da pessoa, levando a uma taxa potencialmente alarmante de falsos positivos com consequente comprometimento ilegal por tempo indeterminado.

g) Vícios comportamentais: essa categoria seria inclusa no novo manual, ao lado de categorias como a seção de vícios em substância. Isso pode se transformar em uma abertura para a criação de uma variedade de diagnósticos tolos como "vícios" de compras, sexo, trabalho, dívidas de cartão de crédito, videogames, etc. Sob a rubrica geral de "vícios de comportamento não especificado", tal categoria representa uma medicalização de escolhas de vida e fornece uma desculpa pronta para a desresponsabilização do sujeito e provavelmente seria usurpada em contextos forenses.

Em dezembro de 2012, a APA publicou um resumo com as modificações oficiais do novo DSM. Além das citadas por Allen Frances, os revisores anunciaram a remoção do sistema multiaxial de diagnósticos. De acordo com a publicação, o DSM-5 combinará os eixos I, II, e III do antigo sistema multiaxial com notaçóes diferentes para os fatores psicossociais e contextuais (antigo eixo IV) e deficiência (antigo eixo V) (APA, 2012).

Foram confirmadas grandes mudanças no diagnóstico de vários transtornos como no que vai ser chamado de "transtorno de espectro autismo". Os novos critérios para o diagnóstico incorporarão vários diagnósticos presentes no DSMIV-TR (APA, 2002). Desse modo, o transtorno de Asperger, o transtorno desintegrativo da infância e o transtorno invasivo do desenvolvimento (não especificado) serão agora categorizados como autismo, o que aumenta a possibilidade de diagnósticos e diminui os meios de tratamento, já que a terapia oferecida é medicamentosa em paralelo com a terapia cognitivo-comportamental.

Será criada, na nova revisão do manual, uma nova sessão para o transtorno de estresse pós-traumático (trauma and stressor-related disorders - TEPT). Assim, o DSM-5 irá focar ainda mais nos sintomas comportamentais que acompanham o TEPT, propondo quatro grupos distintos de diagnóstico. O TEPT também 
será desenvolvido de forma mais sensível para captar os sintomas em crianças e adolescentes.

No transtorno por uso de substâncias, o DSM-5 combinará as categorias do DSM-IV-TR (APA, 2002) de abuso de drogas e dependência de substâncias. Os critérios não só foram combinados como reforçados.

A publicação também informa sobre a inclusão de novos transtornos, tais como o transtorno de acumulação, cuja adição ao DSM-5, de acordo com a publicação, é apoiada pela investigação científica extensiva sobre esse distúrbio. Assim, visa a diagnosticar pessoas com dificuldade de se desfazer de bens, independentemente do seu valor real. $\mathrm{O}$ comportamento geralmente tem efeitos nocivos emocionais, físicos, sociais, financeiros e até mesmo legais para o colecionador e para os membros de sua família; transtorno de escoriação da pele, que será incluído no capítulo distúrbios obsessivo-compulsivos e relacionados; transtorno de aprendizagem específica, que amplia os critérios do DSM-IV-TR (APA, 2002) para representar desordens distintas que interferem na aquisição e utilização de uma ou mais das seguintes habilidades acadêmicas: linguagem oral, leitura, linguagem escrita ou matemática, além de transtornos relacionados a jogos na internet; transtorno do comportamento suicida e transtornos de autoagressão não suicida. No entanto não há mais informações sobre tais transtornos.

As consequências dessa tentativa de predição são graves. Para os sujeitos, incluem tratamento desnecessário com medicamentos que não têm eficácia comprovada, além de apresentarem efeitos colaterais conhecidos, tais como estigma, diminuição da responsabilidade e controle próprio, uma vez que os problemas da vida diária serão medicalizados como transtornos mentais para a melhor adaptação do homem à sociedade moderna.

As novas categorias diagnósticas que virão com o DSM-5 farão parte de um novo capítulo do processo de medicalização da existência, na qual qualquer aspecto da vida do sujeito pode ser considerado um transtorno e:

Consequentemente, deve ser tratado - via medicação - para a não perturbação da ordem pública. Essa patologização da existência revela o novo imperativo da psiquiatria estatística atual: todos transtornados. E se o tratamento privilegiado torna-se farmacológico, que grande negócio para a indústria farmacêutica! (Calazans, Guerra, Kyrillos Neto, Pontes \& Resende, 2012, p. 192).

O que vemos na prática é um retorno das explicações organicistas de transtornos que visam a justificar o mal-estar do sujeito na sociedade moderna. 
Vemos as velhas e falhas tentativas de encontrar no corpo algo que justifique o sintoma, no entanto com uma nova máscara.

Essa tentativa de suprimir o mal-estar está apoiada em parâmetros pseudocientíficos e está caminhando em direção a anular a verdade sobre a angústia. A medicalização da vida propõe eliminar totalmente o sofrimento e vende a garantia de um pretenso bem-estar.

O conceito de medicalização, segundo Aguiar (2004), está ligado à expansão do domínio da profissão médica para outros campos, especialmente aqueles referentes a problemas considerados de ordem espiritual/moral ou legal/criminal. A medicalização é um processo complexo, que envolve não só a criação de novas categorias diagnósticas que orientam para um tratamento farmacológico, mas se apresenta também como uma forte aliada ao processo de intromissão das explicaçôes biológicas no cotidiano das pessoas:

Odiscurso da psiquiatriabiológicavem sendo fortementeveiculado nos meios de comunicação, atingindo todo o tecido social numa produção massificadora de um determinado modo de subjetivação. Cada vez mais, as pessoas incorporam o vocabulário neurocientífico ao modo como experimentam a vida, traduzindo seus próprios sentimentos [...] como "baixa de serotonina", "recaída da depressão", "alteração da dose do antidepressivo", etc. Trata-se da veiculação midiática de um discurso que produz subjetividades "medicalizadas" (p. 137).

O principal desencadeador dessa medicalização é o fato de que os DSM deixam de ter um uso exclusivamente clínico. Desse modo, ele pretende ser usado não somente por psiquiatras na sua prática clínica, mas também em contextos jurídicos, escolares, etc.

No entanto sabemos que a clínica é o lugar de excelência da psicopatologia. George Lantéri-Laura (1989) diz que "não há nada na psicopatologia que não tenha estado antes na clínica” (p. 18). A tensão entre a singularidade da experiência e a universalidade do saber, ou melhor, entre o singular e o geral, própria da clínica é desconsiderada pelo DSM. Onde há um método estatístico que quantifica os sintomas, não há clínica, uma vez que a estatística trata de populações e não de sujeitos.

Não obstante, temos a recusa da "doença mental" no DSM, já que, para operar nessa classificação, seria necessário a presença do fator etiológico. A etiologia é conhecida a partir do momento em que há a especificidade de um marcador biológico (Aguiar, 2004) que explique a causa dos sintomas observados. E quando não se consegue especificar um marcador biológico? Somente resta, então, apelar 
para o conceito de síndrome. Desse modo, o DSM, preventivamente, afirma o aspecto sindrômico dos transtornos mentais. E mais, se não há um marcador biológico que proporcione uma explicação etiológica da doença, por que então temos a direção de um tratamento essencialmente farmacológico a partir do diagnóstico proposto pelo DSM?

\section{Considerações finais}

Podemos dizer também que o projeto da psiquiatria, mesmo com a criação de manuais e o desenvolvimento exponencial de psicotrópicos, ainda hoje não conseguiu atingir sua meta: ser uma ciência médica. São poucos os ditos transtornos que tem comprovadamente uma causa fisiológica, restringindo a aplicação apenas a casos de demência, de deficiência mental e às psicoses orgânicas e sintomáticas.

Tais mudanças compravam mais uma vez que a lógica de transtorno do DSM está pautada numa disfunção pessoal baseada no desvio de uma norma social, uma alteração da ordem que deve ser restituída.

Um exemplo de que o DSM se pauta em valores culturais para estabelecer o que é ou não doença reside no fato de que, em suas primeiras versões, a homossexualidade era tida como transtorno. Hoje, a homossexualidade, que antes era doença e necessitava de um tratamento, não é mais um transtorno. No entanto, o tabagismo, hábito que antes representava prestígio social, atualmente, devido a mudanças dos hábitos culturais, é tido como um transtorno. E são vários os transtornos ligados à nicotina: transtorno por uso de nicotina, dependência de nicotina, transtorno induzido por nicotina, abstinência de nicotina e transtornos relacionados à nicotina sem outra especificação (Dunker \& Kyrillos, 2010).

A questão se que coloca é a seguinte: será necessário medicar quantas pessoas para que tais categorias caiam no esquecimento? 


\section{Referências}

American Psychiatric Association (1980). Diagnostic and Statistical Manual of Mental Disorders: DSM-III. Washington: APA.

American Psychiatric Association (2002). Diagnostic and Statistical Manual of Mental Disorders: DSM-IV-TR. Washington: APA.

American Psychiatric Association (2012). American Psychiatric Association Board of Trustees Approves DSM-5: diagnostic manual passes major milestone before May 2013 publication. Washington: APA. Recuperado a partir de http://totallyadd. com/wp-content/uploads/DSMIV.pdf.

American Psychiatris Association (2013). Diagnostic and Statistical Manual of Mental Disorders, Fifth Edition. Washington: APA.

Aguiar, A. (2004). A psiquiatria no divã. Rio de Janeiro: Relume Dumará.

Andrade, L. H., Wang, Y. P., Andreoni, S., Silveira, C. M., Alexandrino-Silva, C., Siu, E. R., . . Viana, M. C. (2012). Mental disorders in megacities: findings from the São Paulo megacity mental health survey, Brazil. Recuperado a partir de http://journals.plos.org/plosone/article?id=10.1371/journal.pone.0031879

Brasil. Ministério da Saúde. (2008). Clipping: 26 a 28 de dezembro de 2009 sábado, domingo e segunda. Recuperado a partir de http://portal.saude.gov.br/ portal/arquivos/pdf/clipping26272812.pdf.

Brasil. Ministério da Saúde (2011). Boletim de Farmacoepidemiologia, 2. Recuperado a partir de http://www.anvisa.gov.br/sngpc/boletins/2011/ boletim_sngpc_2edatualizada.pdf

Calazans, R., Guerra, A., Kyrillos Neto, F., Pontes S. \& Resende, M. (2012). Manifesto de São João del-Rei em prol de uma psicopatologia clínica. In: F. Kyrillos \& R. Calazans (Orgs.). Psicopatologia em debate: controvérsias sobre os DSM's. (pp. 183-200). Barbacena: Ed. UEMG.

Conselho Federal de Psicologia (2012). Campanha "não à medicalização da vida”. Brasília: CFP. Recuperado a partir de: http://site.cfp.org.br/wp-content/ uploads/2012/07/Caderno_AF.pdf.

Dunker, C. \& Kyrillos Neto, F. (2010, junho). Curar a homossexualidade? A Psicopatologia Prática do DSM no Brasil. Revista Mal-estar e Subjetividade, 10 (2), 425-446. 
Foucault, M. (1988). História da sexualidade: a vontade de saber. (Vol. 1, 17a ed.). Rio de Janeiro: Graal.

Frances, A. (2010). Opening Pandora's box: the 19 worst suggestions for DSM-5. Psychiatric Times. Recuperado a partir de http://www.psychiatrictimes.com/ print/article/10168/1522341.

Hansen, J. H. (2004). Como entender a saúde na comunicação? São Paulo: Paulus.

Jerusalinsky, A. \& Fendrik, S. (Org.). (2011). O livro negro da psicopatologia contemporânea. São Paulo: Via Lettera.

Lanaspa, C.R. (2014). Manifiesto a favor de una psicopatología clinica, que no estadistica. Entrevista concedida a Infocopha. Recuperado a partir de http:// stopdsm.blogspot.com.br.

Lantéri-Laura, G. (1989). Prefácio. In: P. Bercherie. Os fundamentos da clínica. (pp. 13-19). Rio de Janeiro: Jorge Zahar.

Luccheli, J. P. (2007). Le DSM-V et lês troubles de la personnalité. Mental, 19, 78-82.

Organização Mundial de Saúde (1946). Carta da Organização Mundial de Saúde. Recuperado a partir de http://www.direitoshumanos.usp.br/index.php/OMSOrganiza\%C3\%A7\%C3\%A3o-Mundial-da-Sa\%C3\%BAde/constituicao-daorganizacao-mundial-da-saude-omswho.html. 